\title{
Chestnut-like $\mathrm{SnO}_{2} / \mathrm{C}$ Nanocomposites with Enhanced Lithium Ion Storage Properties
}

Lie Yang ${ }^{\mathrm{a}}$, Tao Dai ${ }^{\mathrm{a}}$, Yuecun Wang ${ }^{\mathrm{a}}$, Degang Xie ${ }^{\mathrm{a}}$, R. Lakshmi Narayan ${ }^{\mathrm{a}, \mathrm{b}}, \mathrm{Ju} \mathrm{Li}^{\mathrm{a}}$ c,", Xiaohui Ning ${ }^{\mathrm{a}, *}$

${ }^{a}$ Center for Advancing Materials Performance from the Nanoscale (CAMP-Nano), State Key Laboratory for Mechanical Behavior of Materials, Xi'an Jiaotong University, Xi'an 710049, P. R. China.

${ }^{b}$ Department of Materials Science and Engineering, Carnegie Mellon University, Pittsburgh, Pennsylvania 15213, USA.

${ }^{c}$ Department of Nuclear Science and Engineering, and Department of Materials Science and Engineering, Massachusetts Institute of Technology, Cambridge, Massachusetts 02139, USA.

"Corresponding authors. E-mail addresses: xiaohuining@mail.xjtu.edu.cn (X.H. Ning), liju@mit.edu (J.Li)

\begin{abstract}
Chestnut-like $\mathrm{SnO}_{2}$ and $\mathrm{SnO}_{2} / \mathrm{C}$ nanocomposites with hierarchical structures are synthesized by hydrothermally oxidizing Sn nanoparticles in glucose solution. Structural characterizations using SEM and TEM reveal that the $\mathrm{SnO}_{2}$ nanoparticles are composed of numerous, randomly arranged $\mathrm{SnO}_{2}$ nanosheets with hollow cores. Owing to the short electron and ion diffusion distances and transformation strain accommodation mechanism of this structure, the new $\mathrm{SnO}_{2} / \mathrm{C}$ nanocomposites exhibit enhanced lithium ion storage properties with a capacity of $\sim 930 \mathrm{~mA} \mathrm{~h} \mathrm{~g}^{-1}$ and capacity retention of about $96 \%$ after 100 cycles at $0.1 \mathrm{C}$. An in situ TEM study of the electrochemically driven lithiation/delithiation of $\mathrm{SnO}_{2} / \mathrm{C}$ nanocomposites reveals that their enhanced cycling stability is mainly facilitated by the limited volume expansion and excellent mechanical robustness of the hollow chestnuts.
\end{abstract}

\section{Keywords}

hierarchical hollow structure; $\mathrm{SnO}_{2}$ sheet; anode; lithium ion battery; in situ TEM.

\section{Introduction}

With the rapid development of portable electronic devices, electric vehicles and stationary power storage, lithium ion batteries (LIBs) are now required to have a higher energy density and longer cycle life $[1,2]$. In that context, $\mathrm{SnO}_{2}$ has been identified as a promising anode material and has garnered a lot of attention, prompting many studies on its application prospects. However, while $\mathrm{SnO}_{2}$ possesses a theoretical capacity of $782 \mathrm{~mA} \mathrm{~h} \mathrm{~g}^{-1}[3,4]$, a value which is nearly twice that of commercially used graphite, it undergoes severe capacity fade during charge/discharge cycles $[5,6]$, thereby hindering its practical application.

The underlying cause for capacity fade, as revealed by transmission electron microscope (TEM) observations [7, 8] and X-ray tomography [9] studies is that, $\mathrm{SnO}_{2}$ undergoes a large volume expansion/contraction cycle during the lithiation/delithiation process, leading to its fracture and 
pulverization. Post fracture, the freshly formed $\mathrm{SnO}_{2}$ surfaces continuously participate in side reactions with the electrolyte and get covered with an insulating solid electrolyte interphase (SEI) [10]. This manifests as a loss of electrical contact between the pulverized $\mathrm{SnO}_{2}$ particles and the current connecter, thus triggering capacity fade. To solve this problem, various types of nanosized $\mathrm{SnO}_{2}$ composites such as nanoparticles [11], nanosheets [12-15], nanowires [16], nanotubes [17], hollow particles [18], porous particles [19], core shell particles [20], yolk shell particles [21] and hierarchical hollow particles [3, 22, 23], have been synthesized. Owing to their clever structural design and nanoscale dimensions, these composites exhibit enhanced cycling stability. In particular, the use of hierarchical hollow $\mathrm{SnO}_{2} / \mathrm{C}$ nanoparticles is enticing because of shorter electron and ion diffusional hopping distances and buffer volumes to accommodate transformation strains. However, since they are synthesized on hard templates, like silica [24] or polystyrene (PS) [3, 22] particles, an additional post-processing step for the removal of these templates is essential, which increases the cost. Moreover, during synthesis, a high degree of precision and control needs to be exercised while fixing the $\mathrm{pH}$ values of the reactants [12, 22]. Hence, these multiple complexities restrict the mass production of hierarchical hollow $\mathrm{SnO}_{2} / \mathrm{C}$ to some extent.

To alleviate these production issues, we developed a facile hydrothermal method to prepare hierarchical hollow $\mathrm{SnO}_{2} / \mathrm{C}$ particles with a structure similar to that of the shell of a chestnut. The as-synthesized chestnut-like $\mathrm{SnO}_{2} / \mathrm{C}$ particles exhibit improved lithium storage properties and capacity retention when used as an anode for lithium ion batteries. We study the growth mechanism of these particles by extracting the reaction products at specific intervals and observing them under the scanning electron microscope (SEM) and transmission electron microscope (TEM). Finally, we conduct real-time lithiation/delithiation experiments on the particles inside a TEM and observe morphological changes that explain their enhanced performance as a battery material.

\section{Experimental section}

\section{Sample preparation}

The schematic in Fig. 1a shows the preparation process for chestnut-like $\mathrm{SnO}_{2} / \mathrm{C}$ nanocomposites. First, Sn nanoparticles (NPs) were prepared by reducing $\mathrm{SnCl}_{2}$ with $\mathrm{NaBH}_{4}$ [25]. For this, $0.40 \mathrm{~g}$ polyvinyl pyrrolidone (PVP, K30, AR, Aladdin) was dissolved in $90 \mathrm{~mL}$ diethylene glycol (DEG, AR, Aladdin) by magnetic stirring and ultrasound agitation. This solution was then heated up to 90 ${ }^{\circ} \mathrm{C}$ in an oil bath following which, $0.95 \mathrm{~g} \mathrm{SnCl}_{2}$ (AR, Aladdin) was added to it under magnetic stirring. After the $\mathrm{SnCl}_{2}$ salts completely dissolved, $0.87 \mathrm{~g} \mathrm{NaBH}_{4}$ (AR, Aladdin), dissolved in 8.0 $\mathrm{mL}$ DEG, was added into the solution drop wise in the span of 10 minutes with accompanied stirring. The solution was subsequently cooled to ambient temperature, centrifuged at $9000 \mathrm{r} \mathrm{min}^{-1}$, washed with ethanol and water and air-dried at $80{ }^{\circ} \mathrm{C}$ for several hours.

The following describes how these as-prepared Sn nanoparticles were hydrothermally treated in glucose solution to obtain chestnut-like $\mathrm{SnO}_{2}$ nanoparticles. First, $0.10 \mathrm{~g}$ as-prepared $\mathrm{Sn}$ NPs and $0.10 \mathrm{~g}$ glucose (AR, Aladdin) were dispersed and dissolved into $20 \mathrm{~mL}$ distilled water by ultrasound agitation for 30 minutes. Then, the suspension was transferred into a $100 \mathrm{~mL}$ Teflon-lined stainless steel autoclave and kept in an electric oven at $180{ }^{\circ} \mathrm{C}$ for 20 hours. Light yellow products were collected by centrifugation, water washing, and air drying at $80{ }^{\circ} \mathrm{C}$. Finally, $\mathrm{SnO}_{2}$ nanoparticles were obtained by further heating the light yellow products at $400{ }^{\circ} \mathrm{C}$ for 2 hours in air with a heating rate of $1{ }^{\circ} \mathrm{C} \min ^{-1}$. 
To impart a carbon coating to these particles, a uniform mixture of oleic acid (AR, Aladdin) and as-prepared $\mathrm{SnO}_{2}$ particles with mass ratio of $2.5: 1$ was calcined at $400{ }^{\circ} \mathrm{C}$ under Ar for 2 hours at a ramping rate of $1{ }^{\circ} \mathrm{C} \mathrm{min}^{-1}$.

\section{Morphological and structural characterization}

The morphologies of samples were characterized with a field emission scanning electron microscope (SEM, Hitachi SU6600). The inner structure and chemical composition of the samples was investigated with a transmission electron microscope (TEM, JEOL 2100F, accelerating voltage of $200 \mathrm{kV}$ ), which is equipped with energy-dispersive X-ray spectroscopy (EDS). X-ray diffraction (XRD) measurements were carried out with a Bruker D8 Advance diffractometer to study the crystalline structure of the samples.

\section{Electrochemical measurements}

To assess the lithium ion storage capability of as-prepared $\mathrm{SnO}_{2} / \mathrm{C}$, it was assembled as a working electrode of CR2032 coin cells. The $\mathrm{SnO}_{2} / \mathrm{C}$ electrode was prepared by mixing chestnut-like $\mathrm{SnO}_{2} / \mathrm{C}$ particles $(80$ wt $\%$ ) with 10 wt $\%$ conductive carbon black (Super-P) and 10 wt $\%$ poly(vinylidene fluoride) (PVDF, AR, Aladdin) binder in N-methyl-2-pyrrolidone (NMP, AR, Aladdin) solvent. Subsequently, the obtained slurry was coated on copper foil and dried at $80{ }^{\circ} \mathrm{C}$ for 24 hours. The mass loading was controlled to $\sim 0.5 \mathrm{mg} \mathrm{cm}^{-2}$. Pure lithium foils were used as both the working and reference electrode. The electrolyte was $1.0 \mathrm{M} \mathrm{LiPF}_{6}$ dissolved in a mixture of ethylene carbonate (EC) and diethyl carbonate (DEC) with a volume ratio of EC/DEC=1:1. This cell was assembled in an Ar-filled glove box (Unilab, MBraun) where the concentrations of moisture and oxygen were maintained below $0.1 \mathrm{ppm}$. Galvanostatic charge-discharge performance tests were carried out with a battery tester (BST8-MA, MTI) at various current densities between 0.005 and $2.50 \mathrm{~V}$. Cyclic voltammetry $(\mathrm{CV})$ measurements were performed at a scanning rate of $0.5 \mathrm{mV} \mathrm{s}^{-1}$ within a potential window of $0-2.5 \mathrm{~V}$ on a $\mathrm{CHI} 660 \mathrm{E}$ electrochemical workstation (Shanghai Chenhua). It should be noted that the total weight of $\mathrm{SnO}_{2}$ and the carbon layer $\left(\mathrm{SnO}_{2} / \mathrm{C}\right)$ was used to calculate the specific capacity values.

\section{In situ TEM observation}

The in situ TEM characterization was carried out in an environmental transmission electron microscope (ETEM, Hitachi H9500, $300 \mathrm{kV}$ ) using the PI $95 \mathrm{H1H}$ holder (Hysitron). An all-solid nanobattery was assembled. The cluster of chestnut-like $\mathrm{SnO}_{2} / \mathrm{C}$ nanocomposites attached to an aluminum plate with conductive silver epoxy was set as the working electrode, and Li metal loaded on a tungsten tip was set as the counter electrode. $2 \mathrm{~Pa} \mathrm{CO}_{2}$ was pumped into the ETEM chamber which reacted with the naturally formed $\mathrm{Li}_{2} \mathrm{O}$ surface layer on $\mathrm{Li}$ metal to form $\mathrm{Li}_{2} \mathrm{CO}_{3} \cdot \mathrm{Li}_{2} \mathrm{CO}_{3}$ served as the solid electrolyte and was much more stable under electron beam irradiation than the widely used $\mathrm{Li}_{2} \mathrm{O}$ [26]. A bias potential of $-4.0 \mathrm{~V}$ was applied to the chestnut-like $\mathrm{SnO}_{2} / \mathrm{C}$ nanocomposites to initiate the lithiation, while the bias was reversed to facilitate delithiation. More than 10 fully reversed cycles of bias potential were employed to test the cycling stability of these particles.

\section{Results and discussions}

Sn nanoparticles, the precursors in the synthesis of $\mathrm{SnO}_{2} / \mathrm{C}$ nanocomposites, are fully dense spheres with an average diameter of about $110 \mathrm{~nm}$ (See Fig. S1a-b in Supplementary Information (SI)). The XRD results shown in Fig. 1b (red curve), confirm that they exist in the form of pure 
crystalline tetragonal white tin. After these Sn particles are oxidized in glucose solution during the hydrothermal treatment, hierarchical hollow nanoparticles are produced (See Fig. S1c-d). XRD patterns in Fig. $1 \mathrm{~b}$ (blue curve) reveal that the oxidized nanoparticles are tetragonal rutile $\mathrm{SnO}_{2}$. The shell of an individual $\mathrm{SnO}_{2}$ nanoparticle is composed of many randomly arranged nanosheets, as shown in the magnified SEM image in Fig. 2a. Since the structural morphology of these particles is similar to that of a 'chestnut', shown as a reference in Fig. 2b, these $\mathrm{SnO}_{2}$ nanoparticles will hereafter be referred to as 'chestnut-like nanoparticles'. These unique structures contain multiple layers of $\mathrm{SnO}_{2}$ nanosheets and vast inner void spaces which bestow them with high specific surface area and large buffer volume. A conductive carbon layer is coated on the surface of these chestnut-like nanoparticles to improve their electrical conductivity. A high resolution TEM (HRTEM) image of the edge of a chestnut-like $\mathrm{SnO}_{2} / \mathrm{C}$ particle in Fig. 2c shows that a compact amorphous carbon layer, measuring $5-10 \mathrm{~nm}$ in thickness, is formed. In addition to imparting a high quality carbon coat, the chestnut-like structure of the obtained $\mathrm{SnO}_{2} / \mathrm{C}$ particle remains unaffected (see Fig. S1e-f of SI) by the coating treatment.

a

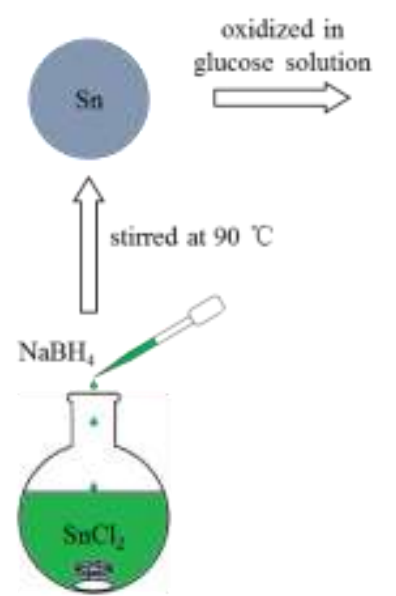

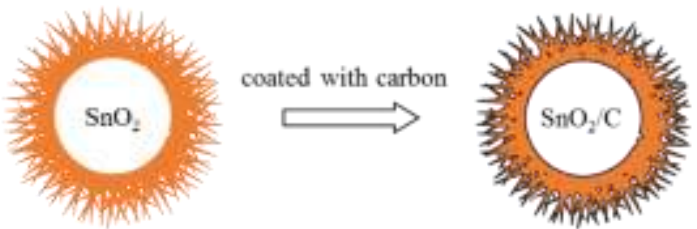

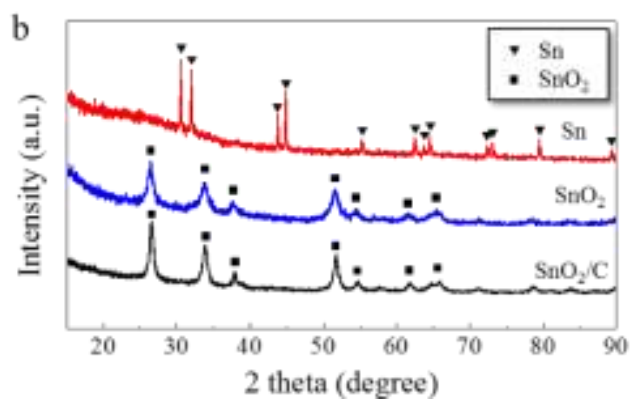

Fig. 1 (a) Schematic illustration of the preparation process for chestnut-like $\mathrm{SnO}_{2} / \mathrm{C}$ nanocomposites; (b) corresponding XRD patterns of the products obtained at each step.
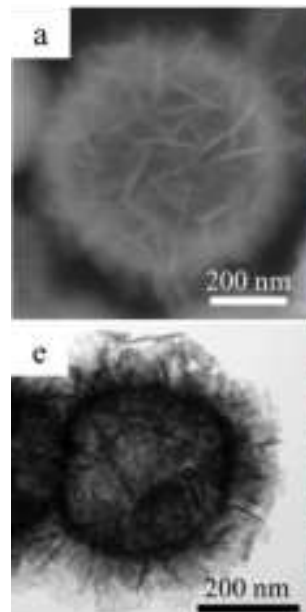
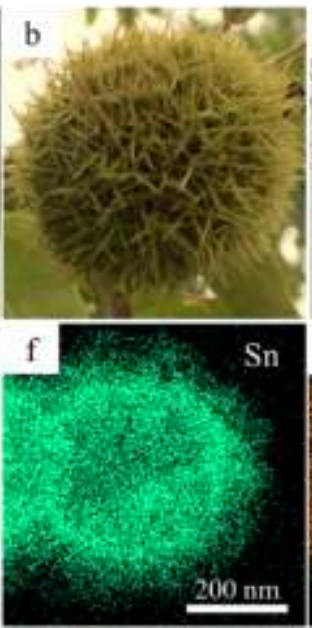

c Nex/ $\mathrm{d}$
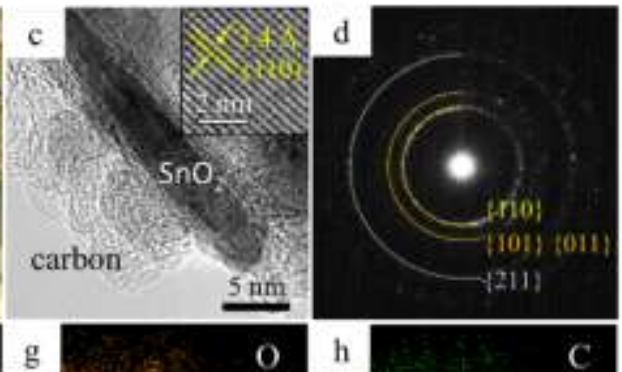

o

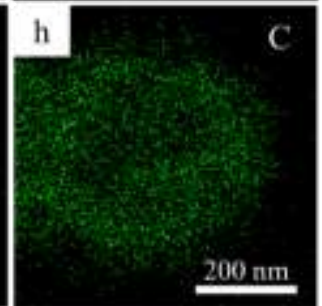

Fig. 2 Morphological and compositional characterizations. (a) SEM image of a single $\mathrm{SnO}_{2}$ particle; (b) photograph of a chestnut seed (Castanea); (c) high resolution TEM (HRTEM) image of the surface of a $\mathrm{SnO}_{2} / \mathrm{C}$ particle. The inset shows the lattice image of $\mathrm{SnO}_{2}$ nanosheet; (d) SAED pattern of a single $\mathrm{SnO}_{2} / \mathrm{C}$ particle; (e) bright field (BF) scanning transmission electron microscope (STEM) 
image of a single $\mathrm{SnO}_{2} / \mathrm{C}$ particle; (f-h) $\mathrm{Sn}, \mathrm{O}$, and $\mathrm{C}$ elements mapping of the $\mathrm{SnO}_{2} / \mathrm{C}$ particle shown in (e).

Beneath the carbon layer, $\mathrm{SnO}_{2}$ exists in the crystalline state as seen from the XRD pattern shown in Fig. 1b (black curve). Also, a lattice spacing of $3.4 \AA$ measured from the inset of Fig. 2 c matches well with the (110) plane of rutile $\mathrm{SnO}_{2}$, thereby suggesting that the preferential growth direction of $\mathrm{SnO}_{2}$ nanosheets is [110]. The selected area electron diffraction (SAED) pattern shown in Fig. 2d further confirms the polycrystalline nature of the chestnut-like $\mathrm{SnO}_{2} / \mathrm{C}$ composites. The energy-dispersive X-ray spectroscopy (EDS) element mapping of the composite nanoparticle in Fig. 2e reveals that the spatial distribution of carbon (Fig. 2h) superimposes perfectly over that of $\mathrm{SnO}_{2}$ (Fig. 2f-g), thus suggesting an intimate contact between the two materials.

To understand the transformation of chestnut-like $\mathrm{SnO}_{2}$ nanoparticles from the initial spherical solid Sn nanoparticles, samples are extracted from the reaction mix at different times. In Fig. 3a-h, the SEM and TEM images of the typical products obtained after a reaction time of 0 hour, 3 hours, 5 hours and 20 hours, respectively are shown. At 0 hour, or as the initial condition before oxidation in glucose solution, the pristine $\mathrm{Sn}$ nanoparticle is a spherical solid with a smooth surface as seen in Fig. 3a-b. However, after 3 hours the surface gets a rough appearance (see Fig. 3c-d) owing to numerous nano-protrusions appearing around the surface of the nanoparticles. Around the same time, the inner Sn core starts shrinking, such that the solid particle now gets a shell-like structure. When the reaction time progresses to 5 hours, the Sn core shrinks further and a few tiny sheets of $\mathrm{SnO}_{2}$ could be observed on the surface of the particle (refer to Fig. 3e-f). Finally, after 20 hours, the core is completely consumed and a chestnut-like hierarchical hollow structure is obtained (see Fig. $3 g-h)$.

Additionally, during the transformation of chestnut-like $\mathrm{SnO}_{2}$, the phase evolution of these nanoparticles is also simultaneously tracked using XRD. It is observed that as the reaction time increases, the intensity of peaks representing tetragonal white $\mathrm{Sn}$ decreases gradually, thus suggesting that the pristine Sn nanoparticles are getting oxidized continuously. This inference is consistent with the observation of shrinkage and consumption of Sn cores seen in Fig. 3c-h. The appearance of characteristic peaks of both $\mathrm{SnO}$ and $\mathrm{SnO}_{2}$ at 3 hours and 5 hours suggests a possible two-step oxidation of Sn nanoparticles under this hydrothermal condition. However, after 20 hours, XRD only shows characteristic peaks of $\mathrm{SnO}_{2}$ implying that all the $\mathrm{Sn}$ nanoparticles are eventually oxidized to $\mathrm{SnO}_{2}$.

Based on the above described structural and compositional changes, the transformation of $\mathrm{Sn}$ nanoparticles into hollow structures can be explained as the following. In the initial stages, the outer surfaces of $\mathrm{Sn}$ nanoparticles are oxidized by reacting with water: $\mathrm{Sn}+\mathrm{H}_{2} \mathrm{O}=\mathrm{SnO}+\mathrm{H}_{2}$. Now, due to the difference in diameters and charge states of the ions, the outward diffusion of $\mathrm{Sn}$ ions is much faster than that of oxygen ions diffusing inwards [27]. Therefore, to balance the net effect of outward diffusion, there is a net inward flux of vacancies. These out-of-equilibrium vacancies accumulate into voids that merge into a large void sheath. Such a voiding mechanism was previously observed in $\mathrm{ZnO}_{2}$ and $\mathrm{CoO}$ nanoparticles and is understood as an extreme limit of the Kirkendall effect $[17,28,29]$. The temperature of the hydrothermal treatment $\left(180^{\circ} \mathrm{C}\right)$ is very close to the melting point of metallic tin $\left(232{ }^{\circ} \mathrm{C}\right)$, so surface/interfacial diffusion of $\mathrm{Sn} / \mathrm{Sn}^{x+}$ should be operative. The residual Sn core still contacts the oxide at points due to adhesion, providing $\mathrm{Sn}$ source for oxide growth. With time, the Sn cores get fully consumed by the growing voids and 
eventually result in spherical hollow structures.

To understand how randomly arranged $\mathrm{SnO}_{2}$ nanosheets form on the outer surface of the $\mathrm{SnO}_{2}$ particle, we synthesized a batch of particles without adding glucose to the reaction mix. When we compare the final product obtained with and without the addition of glucose (see Fig. S2), it was noted that the former imparts a chestnut-like morphology while the latter preserves the initial spherical shape of the Sn particle. This implies that the presence of glucose plays a crucial role in the formation of the randomly arranged nanosheets. Previous studies have shown that glucose is hydrolyzed into small functional groups under hydrothermal conditions [30, 31]. Since such functional groups should also form in our synthesis, we believe that they may get absorbed on some specific lattice planes of $\mathrm{SnO}_{2}$ and suppress crystal growth along certain directions. Therefore, nanosheets form only along certain preferred directions and from HRTEM images, the plane normal was determined to be the [110] direction.

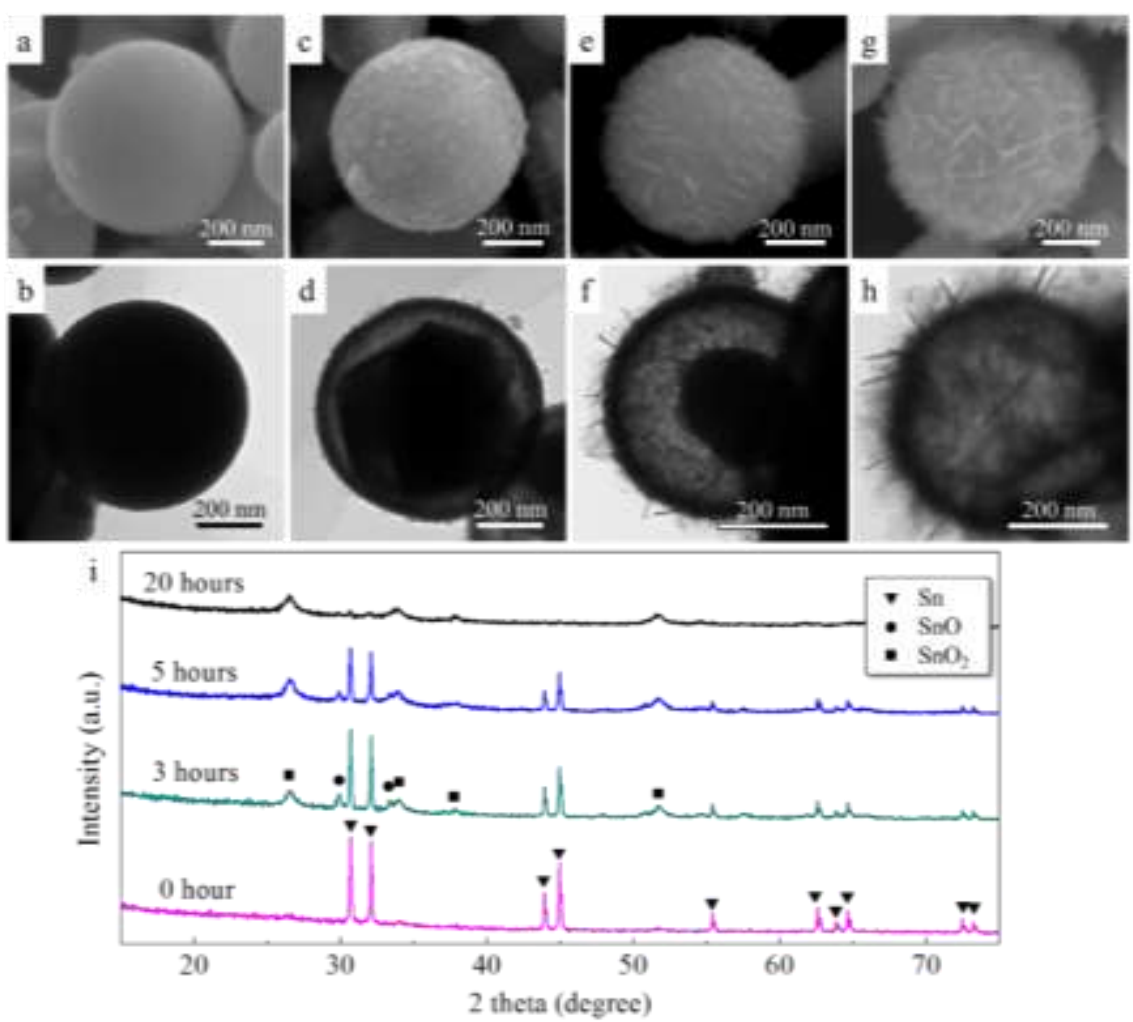

Fig. 3 Ex situ SEM and TEM observation of typical $\mathrm{SnO}_{x}$ particles obtained at increasing reaction time: (a-b) 0 hour, (c-d) 3 hours, (e-f) 5 hours, (g-h) 20 hours; (i) corresponding XRD patterns of $\mathrm{SnO}_{x}$ particles.

From the preceding discussion it is evident that our method of synthesizing $\mathrm{SnO}_{2}$ nanoparticles is less complicated and uses lesser number of reactants (see table S1) compared to other methods that require hard templates and precise $\mathrm{pH}$ control $[12,15,22,23]$. In the following paragraphs, we will look at the advantages of chestnut-like $\mathrm{SnO}_{2} / \mathrm{C}$ nanocomposites possessing unique structural attributes like high specific surface area, large void space and highly conductive surface coating. These features will be discussed in the context of designing and utilizing chestnut-like $\mathrm{SnO}_{2} / \mathrm{C}$ nanoparticles as anode materials for LIBs.

Variations of current, $\mathrm{mA}$, and potential, $\mathrm{V}$, obtained from the cyclic voltammetry experiments of the present nanocomposites is displayed in Fig. 4a. In this plot, the strong cathodic peak observed at 
$0.55 \mathrm{~V}$ in the first cycle can be attributed to the reduction of $\mathrm{SnO}_{2}$ to $\mathrm{Sn}$, as described by Eq. 1 [32], and the formation of a solid electrolyte interphase (SEI) layer. And, the current peak at $0.01 \mathrm{~V}$ corresponds to an alloying process that forms $\mathrm{Li}_{x} \mathrm{Sn}$ according to Eq. 2 [32].

$$
\begin{gathered}
\mathrm{SnO}_{2}+4 \mathrm{Li}^{+}+4 \mathrm{e}^{-} \rightleftharpoons \mathrm{Sn}+2 \mathrm{Li}_{2} \mathrm{O} \\
\mathrm{Sn}+x \mathrm{Li}^{+}+x \mathrm{e}^{-} \rightleftharpoons \mathrm{Li}_{x} \mathrm{Sn}(0<x<4.4)
\end{gathered}
$$

In the anodic process of the first cycle, the peaks at $0.78 \mathrm{~V}$ and $1.30 \mathrm{~V}$ correspond to the de-alloying of $\mathrm{Li}_{x} \mathrm{Sn}$ and partial reversible formation of $\mathrm{SnO}_{2}$, respectively, as established by previous studies $[33,34]$. However, in the $3^{\text {rd }}$ and $5^{\text {th }}$ cycles, nearly no peaks are observed at $0.55 \mathrm{~V}$, indicating that the formation of SEI is mitigated or even terminated. Additionally, it can be seen that the $\mathrm{CV}$ curves of the $3^{\text {rd }}$ and $5^{\text {th }}$ cycle overlap with each other, therefore suggesting that the electrochemical reactions are fully reversible.

To assess the cycling stability of these materials during charging and discharging, the voltage profiles as a function of specific capacity, at the current density of $0.1 \mathrm{C}$ are shown in Fig. $4 \mathrm{~b}$. Note that the current density of $1.0 \mathrm{C}$ corresponds to $782 \mathrm{~mA} \mathrm{~g}^{-1}$. While the initial discharge capacity is found to be as high as $1600 \mathrm{~mA} \mathrm{~h} \mathrm{~g}^{-1}$, the subsequent charge capacity decreases to $900 \mathrm{~mA} \mathrm{~h} \mathrm{~g}^{-1}$, recording a capacity loss of $\sim 44 \%$. This large capacity loss is mainly caused by the inevitable formation of the SEI layer and incomplete extraction of lithium from the active material. However, in the same figure, it can be seen that the capacity losses are largely reduced subsequent to the second cycle, such that only $0.4 \%$ capacity fade was observed between the $10^{\text {th }}$ cycle and $50^{\text {th }}$ cycle, with a capacity loss rate of only $\sim 10^{-4}$ per cycle, which is extraordinary. Besides, we can see that the curve of the $10^{\text {th }}$ cycle almost overlaps with the curve of the $50^{\text {th }}$ cycle, which again highlights the excellent cycling stability and reversibility of these $\mathrm{SnO}_{2} / \mathrm{C}$ chestnuts.
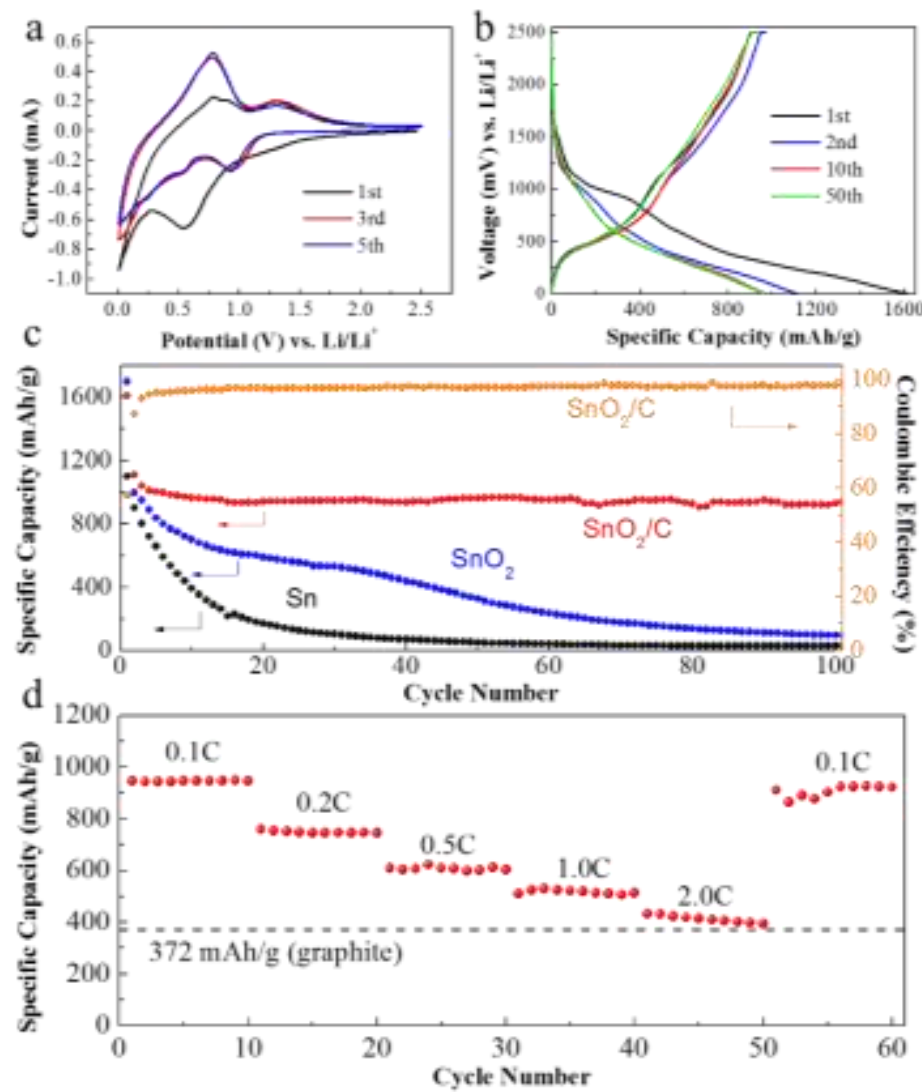

Fig. 4 Electrochemical performance of chestnut-like $\mathrm{SnO}_{2} / \mathrm{C}$ nanocomposites. (a) Representative 
cyclic voltammograms (CV) at a scanning rate of $0.5 \mathrm{mV} \mathrm{s}^{-1}$; (b) charge/discharge voltage profiles for the 1st, 2nd, 10th, and 50th cycles at a current density of 0.1C; (c) cycle performance at a current density of $0.1 \mathrm{C}$ (arrows indicate the reference axes); (d) rate capability of chestnut-like $\mathrm{SnO}_{2} / \mathrm{C}$.

To examine the cycling stability further, the specific capacity of $\mathrm{SnO}_{2} / \mathrm{C}$ is plotted as a function of the number of cycles in Fig. 4c. As can be seen here, $\mathrm{SnO}_{2} / \mathrm{C}$ exhibits a uniform discharge capacity of $930 \mathrm{~mA} \mathrm{~h} \mathrm{~g}^{-1}$ even after 100 cycles. Interestingly, this value is higher than the theoretical capacity of $\mathrm{SnO}_{2}$, which is $\sim 782 \mathrm{~mA} \mathrm{~h} \mathrm{~g}^{-1}$, provided that the only active chemical reaction occurring is the one shown in Eq. 2. Therefore, our $\mathrm{SnO}_{2} / \mathrm{C}$ nanocomposites can display additional capacity only if the reaction shown in Eq. 2 and the partial reversal of the redox reaction described in Eq. 1[3, 25, 35] occur concurrently. This is confirmed by SAED and XRD characterization of fully charged active particles that exhibit signals of both $\mathrm{Sn}$ and $\mathrm{SnO}_{2}$, as shown in Fig. S3. Note that, the capacity retention ratio of $\mathrm{SnO}_{2} / \mathrm{C}$ is as high as $96 \%$ after 100 cycles, which is significantly higher than those reported in the literature for other $\mathrm{SnO}_{2}$ based hierarchical particles (see Table S2). This enhanced cycling stability can be ascribed to the large void space of chestnut-like structure, which buffers the volume expansion of both the single $\mathrm{SnO}_{2} / \mathrm{C}$ particle and the electrode film, thus maintaining its electron conduction integrity and facilitating the high level utilization of $\mathrm{SnO}_{2}$.

For a more comprehensive evaluation of our $\mathrm{SnO}_{2} / \mathrm{C}$ nanocomposites, the electrochemical behavior of bare $\mathrm{Sn}$ nanoparticles and bare chestnut-like $\mathrm{SnO}_{2}$ are also evaluated by galvanostatic charge/discharge experiments and the results are included in Fig. 4c. It can be seen that compared to the $\mathrm{SnO}_{2} / \mathrm{C}$ particle, both chestnut-like $\mathrm{SnO}_{2}$ particles without carbon and bare $\mathrm{Sn}$ nanoparticles exhibit faster capacity fading rate as their remaining capacity decreases to about $98 \mathrm{~mA} \mathrm{~h} \mathrm{~g}^{-1}$ after 100 cycles and $100 \mathrm{~mA} \mathrm{~h} \mathrm{~g}^{-1}$ after just 30 cycles, respectively. Bare Sn particles display poor cycling stability because their drastic dimensional changes cause SEI instability that disrupts and destroys electron conduction path during cycling. As a result, bare Sn particles are not efficiently utilized if used in the cell reactions. However, as an embedded part of the oxide-metal composite formed after the initial reaction shown in Eq. 1, due to the constraining effect of the surrounding $\mathrm{Li}_{2} \mathrm{O}$ oxide that suppresses SEI instability somewhat, Eq. 2 can proceed reversibly for the embedded Sn particles. On the other hand, the performance difference between chestnut-like $\mathrm{SnO}_{2} / \mathrm{C}$ and $\mathrm{SnO}_{2}$ particles can be ascribed to the coated protective carbon layer which separates $\mathrm{SnO}_{2}$ from the electrolyte, preventing the SEI from forming directly on the surface of $\mathrm{SnO}_{2}$, and making electron conduction paths even more difficult to disrupt. This observation suggests that it is necessary to coat carbon on $\mathrm{SnO}_{2}$ particles to facilitate their superior performance. 

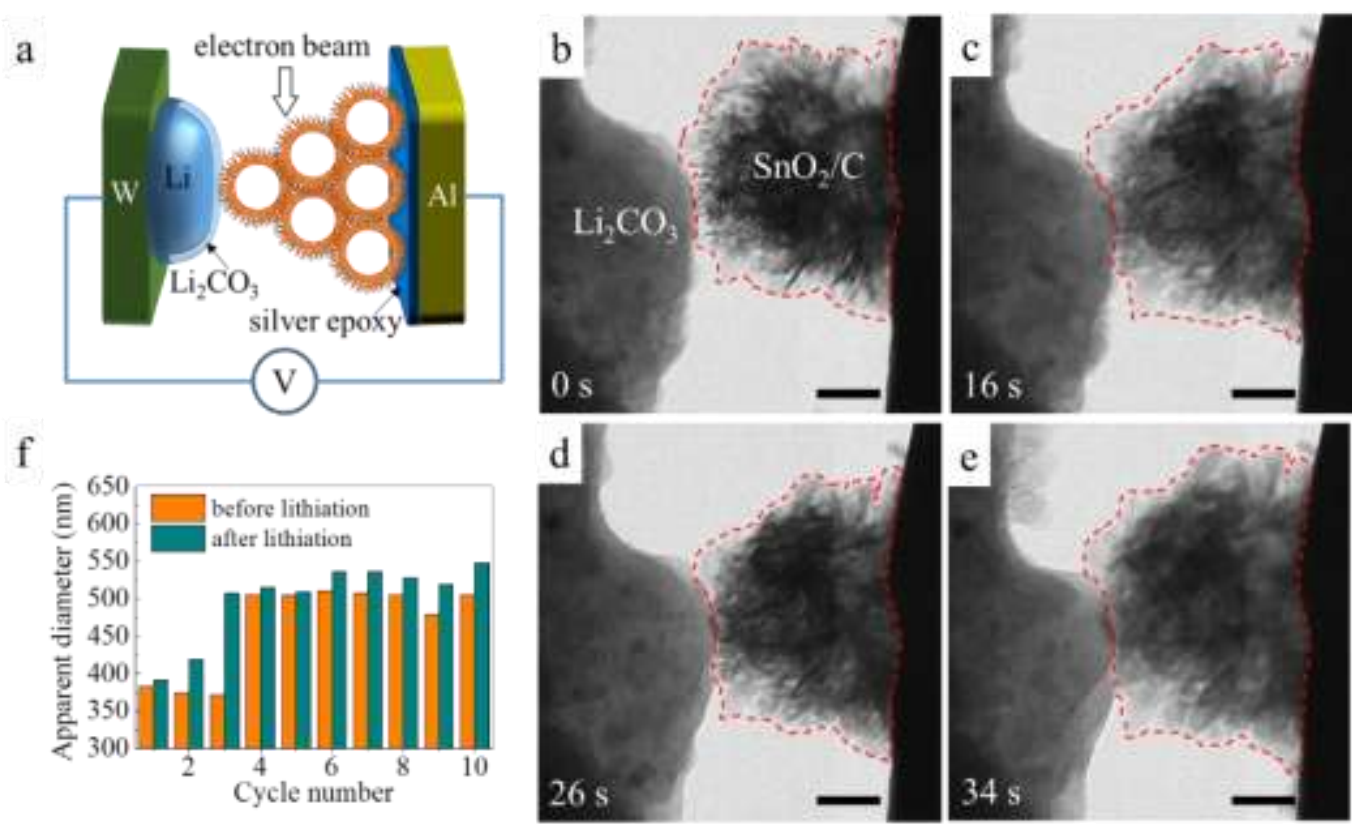

Fig. 5: In situ TEM observation of the morphology evolution of chestnut-like $\mathrm{SnO}_{2} / \mathrm{C}$ nanocomposites during lithiation/delithiation process. (a) schematic illustration for the nanobattery set-up; (b-e) still frames extracted from the recorded movie during $1^{\text {st }}$ and $2^{\text {nd }}$ lithiation/delithiation cycle, (b) pristine cluster, (c) after $1^{\text {st }}$ lithiation, (d) after $1^{\text {st }}$ delithiation, (e) after $2^{\text {nd }}$ lithiation, scale bar: $100 \mathrm{~nm}$; see also Movie S1 to appreciate the accordion-like motion. (f) apparent diameters of $\mathrm{SnO}_{2} / \mathrm{C}$ clusters during cyclic lithiation/delithiation process.

Additionally, as shown in Fig. 4d, chestnut-like $\mathrm{SnO}_{2} / \mathrm{C}$ particles exhibit good rate capability with the discharge capacities of $945,750,610,520$ and $410 \mathrm{~mA} \mathrm{~h} \mathrm{~g}^{-1}$ at the current densities of $0.1 \mathrm{C}, 0.2$ $\mathrm{C}, 0.5 \mathrm{C}, 1 \mathrm{C}$ and $2 \mathrm{C}$, respectively. Remarkably, when the current density was restored to $0.1 \mathrm{C}$ after $2 \mathrm{C}$ testing, about $97.8 \%\left(925 \mathrm{~mA} \mathrm{~h} \mathrm{~g}^{-1}\right)$ of the initial specific capacity was retained, revealing robustness of the $\mathrm{SnO}_{2} / \mathrm{C}$ electrode. It is believed that this good rate capability is associated with the nanostructure which shortens the diffusional hopping distances of lithium ions and electrons within the nanocomposite.

To explain the enhanced stability of the chestnut-like $\mathrm{SnO}_{2} / \mathrm{C}$ particles, in situ TEM measurements were performed to observe the morphological evolution of chestnut-like $\mathrm{SnO}_{2} / \mathrm{C}$ nanocomposites during the lithiation/delithiation process. A schematic illustration of the all-solid nanobattery is shown in Fig. 5a. By biasing $\mathrm{SnO}_{2} / \mathrm{C}$ nanocomposites with a potential of $-4.0 \mathrm{~V}$, lithiation was initiated. As shown in Fig. 5b-e and Movie S1, during the first two cycles, the cluster expanded and contracted reversibly like an accordion, suggesting excellent reversibility. The chestnut-like structure does not develop any cracks, suggesting a high degree of structural integrity during the cyclic lithiation and delithiation. Subsequently, bias potentials of $-4.0 \mathrm{~V}$ and $+4.0 \mathrm{~V}$ were applied repeatedly for ten cycles. During these cycles, the apparent diameter for a particular combination of lithiation and delithiation cycle is quite stable, as seen in Fig. 5f. It should be noted that the sudden increase in the apparent diameter after lithiation in the $3^{\text {rd }}$ cycle derives little contribution from the lithiation process, but is rather a result of the movement of the aluminum current collector, which exposes a higher proportion of $\mathrm{SnO}_{2} / \mathrm{C}$ nanocomposites in the TEM view (See Movie S2). For all individual cycles considered, the calculated increase of apparent diameter 
after lithiation is consistently less than $12 \%$. This value is much lower than that seen for pure $\mathrm{SnO}_{2}$ which shows an increase of $\sim 34 \%$ [36]. Note that fully dense $\mathrm{SnO}_{2}$ nanoparticles can only expand laterally during lithiation. On the other hand, since the hollow core and sheet-configuration of the chestnut-like $\mathrm{SnO}_{2} / \mathrm{C}$ nanoparticles leaves a large buffering space for volume expansion, the medial and lateral movement of the active materials is facilitated. Therefore, the chestnut-like $\mathrm{SnO}_{2} / \mathrm{C}$ nanoparticles undergo smaller apparent volume changes during lithiation/delithiation as seen from the in situ TEM movies and images. Owing to such facial accommodation mechanism of the transformation strains, chestnut-like $\mathrm{SnO}_{2} / \mathrm{C}$ nanocomposites exhibit a remarkable ability to reversibly undergo cyclic electrochemical changes without fatigue induced failure. In summary, by utilizing the in situ TEM technique we demonstrate that the chestnut-like $\mathrm{SnO}_{2} / \mathrm{C}$ nanocomposites exhibit excellent structural integrity and reversibility, remarkable toughness and minimal volume expansion during cyclic lithiation and delithiation process. These attributes are critical for maintaining a high utilization ratio of active $\mathrm{SnO}_{2} / \mathrm{C}$ materials and promote enhanced cycling stability of chestnut-like $\mathrm{SnO}_{2} / \mathrm{C}$ particles as seen from their electrochemical performance.

\section{Conclusions}

We have developed a facile hydrothermal method to synthesize chestnut-like hierarchical hollow $\mathrm{SnO}_{2}$ nanoparticle and $\mathrm{SnO}_{2} / \mathrm{C}$ nanocomposite. The unique burr structure of the $\mathrm{SnO}_{2}$ presents shorter electron and ion diffusional hopping distances and buffer volume, such that the $\mathrm{SnO}_{2} / \mathrm{C}$ nanocomposite shows good electrochemical performance as the anode of a lithium ion battery. These chestnut-like nanocomposites with size of about $400 \mathrm{~nm}$ exhibit high reversible specific capacity of $930 \mathrm{~mA} \mathrm{~h} \mathrm{~g}^{-1}$ and capacity retention of about $96 \%$ after 100 cycles at $0.1 \mathrm{C}$. The in situ study of the electrochemically driven lithiation and delithiation of $\mathrm{SnO}_{2} / \mathrm{C}$ nanocomposites in TEM demonstrated that the apparent volume expansion of $\mathrm{SnO}_{2} / \mathrm{C}$ chestnut during lithiation is less than that of fully dense $\mathrm{SnO}_{2}$, and is highly robust, which contributed to its enhanced cycling stability and remarkable rate capability. Our method of synthesis may be extended to produce other hierarchical oxides as well.

\section{Acknowledgements}

This work was supported by the grants from National Natural Science Foundation of China (51401157) and Basic Science Research in Shaan Xi (2015JQ5164). JL acknowledges support by NSF DMR-1410636.

\section{References}

[1] J. M. Tarascon,M. Armand, Nature 414 (2001) 359-367.

[2] V. Etacheri, R. Marom, R. Elazari, G. Salitra,D. Aurbach, Energy Environ. Sci. 4 (2011) 3243-3262.

[3] J. Liang, X. Y. Yu, H. Zhou, H. B. Wu, S. Ding,X. W. Lou, Angew. Chem. Int. Ed. 53 (2014) 12803-12807.

[4] J. S. Chen,X. W. Lou, Small 9 (2013) 1877-1893.

[5] M. S. Park, G. X. Wang, Y. M. Kang, D. Wexler, S. X. Dou, H. K. Liu, Angew. Chem. Int. Ed. 46 (2007) 750-753. 
[6] S. Ding, J. S. Chen,X. W. Lou, Adv. Funct. Mater. 21 (2011) 4120-4125.

[7] J. Y. Huang, L. Zhong, C. M. Wang, J. P. Sullivan, W. Xu, L. Q. Zhang, S. X. Mao, N. S. Hudak, X. H. Liu, A. Subramanian, H. Fan, L. Qi, A. Kushima,J. Li, Science 330 (2010) 1515-1520.

[8] R. Retoux, T. Brousse,D. M. Schleich, J. Electrochem. Soc. 146 (1999) 2472-2476.

[9] M. Ebner, F. Marone, M. Stampanoni,V. Wood, Science 342 (2013) 716-20.

[10] J. Y. Cheong, J. H. Chang, H. K. Seo, J. M. Yuk, J. W. Shin, J. Y. Lee,I.-D. Kim, Nano Energy 25 (2016) 154-160.

[11] K. Ui, S. Kawamura,N. Kumagai, Electrochim. Acta 76 (2012) 383-388.

[12] H. B. Wu, J. S. Chen, X. W. Lou,H. H. Hng, J. Phys. Chem. C 115 (2011) 24605-24610.

[13] Y. Zhu, H. Guo, H. Zhai,C. Cao, ACS Appl. Mater. Interfaces 7 (2015) 2745-2753.

[14] L. Zhang, H. B. Wu,X. Wen Lou, Mater. Horiz. 1 (2014) 133-138.

[15] C. Wang, Y. Zhou, M. Ge, X. Xu, Z. Zhang,J. Z. Jiang, J. Am. Chem. Soc. 132 (2010) 46-47.

[16] J. Liu, Y. Li, X. Huang, R. Ding, Y. Hu, J. Jiang,L. Liao, J. Mater. Chem. 19 (2009) 1859-1864.

[17] P. Wu, N. Du, H. Zhang, J. Yu, Y. Qi,D. Yang, Nanoscale 3 (2011) 746-750.

[18] S. Ding, J. S. Chen, G. Qi, X. Duan, Z. Wang, E. P. Giannelis, L. A. Archer,X. W. Lou, J. Am. Chem. Soc. 133 (2011) 21-23.

[19] Y. Yu, C. H. Chen,Y. Shi, Adv. Mater. 19 (2007) 993-997.

[20] X. Zhou, Y.-X. Yin, L.-J. Wan,Y.-G. Guo, J. Mater. Chem. 22 (2012) 17456-17459.

[21] Y. J. Hong, M. Y. Son,Y. C. Kang, Adv. Mater. 25 (2013) 2279-2283.

[22] S. Ding,X. Wen Lou, Nanoscale 3 (2011) 3586-3588.

[23] X. M. Yin, C. C. Li, M. Zhang, Q. Y. Hao, S. Liu, L. B. Chen,T. H. Wang, J. Phys. Chem. C 114 (2010) 8084-8088.

[24] X. W. Lou, C. M. Li,L. A. Archer, Adv. Mater. 21 (2009) 2536-2539.

[25] P. Wu, N. Du, H. Zhang, C. Zhai,D. Yang, ACS Appl. Mater. Interfaces 3 (2011) 1946-1952.

[26] X. H. Liu, L. Zhong, S. Huang, S. X. Mao, T. Zhu,J. Y. Huang, ACS Nano 6 (2012) 1522-1531.

[27] J. S. Cho,Y. C. Kang, Small 11 (2015) 4673-4681.

[28] Y. Yin, R. M. Rioux, C. K. Erdonmez, S. Hughes, G. A. Somorjai,A. P. Alivisatos, Science 304 (2004) 711-714.

[29] H. J. Fan, U. Gösele,M. Zacharias, Small 3 (2007) 1660-1671.

[30] G. Bonn,O. Bobleter, J. Radioanal. Chem. 79 (1983) 171-177.

[31] D. Klingler,H. Vogel, J. of Supercritical Fluids 55 (2010) 259-270.

[32] I. A. Courtney,J. R. Dahn, J. Electrochem. Soc. 144 (1997) 2045-2052.

[33] Z. Wang, Z. Wang, S. Madhavi,X. Wen Lou, J. Mater. Chem. 22 (2012) 2526-2531.

[34] C. Zhu, S. Zhu, K. Zhang, Z. Hui, H. Pan, Z. Chen, Y. Li, D. Zhang,D.-W. Wang, Sci. Rep. 6 (2016) 25829.

[35] S.-Y. Lee, K.-Y. Park, W.-S. Kim, S. Yoon, S.-H. Hong, K. Kang,M. Kim, Nano Energy 19 (2016) 234-245

[36] X. H. Liu,J. Y. Huang, Energy Environ. Sci. 4 (2011) 3844-3860. 